\title{
Transient Small-Bowel Intussusception Unmasking Adult Celiac Disease
}

\author{
Maryam AlAhmad ${ }^{a} \quad$ Abdulqader Almessabi $^{b}$ \\ ${ }^{a}$ Gastroenterology Fellow, Sheikh Shakhbout Medical City, Abu Dhabi, UAE; \\ ${ }^{\mathrm{b}}$ Gastroenterology Consultant, Sheikh Shakhbout Medical City, Abu Dhabi, UAE
}

\section{Keywords}

Celiac disease $\cdot$ Intussusception $\cdot$ Tissue transglutaminase antibodies · Diarrhea $\cdot$ Abdominal pain

\begin{abstract}
Celiac disease $(C D)$ is a chronic immune-mediated small-intestinal disorder. The tissue transglutaminase (TTG) antibodies test is the initial step in the diagnosis of CD. Intussusception is a process where one segment of the bowel invaginates into the lumen of an adjacent segment. Despite that, the association between adult intussusception and CD has been descried previously, and it is still not widely recognized. We report a case of adult intussusception as the first manifestation of CD. A 28-year-old nonalcoholic and nonsmoker, previously healthy man, presented to the emergency department with a 1-week history of progressive diffuse abdominal pain associated with abdominal distension, nausea, vomiting, diarrhea, and $7 \mathrm{~kg}$ weight loss. The physical exam was remarkable for thin body habitus with no lymphadenopathy. Laboratory testing was significant for hypochromic microcytic anemia, AST 214 IU/L, ALT $217 \mathrm{IU} / \mathrm{L}$, alkaline phosphatase $183 \mathrm{IU} / \mathrm{L}$, and INR 2.9. An abdominal radiograph showed distended gas-filled small- and large-bowel loops. An abdominal computed tomography scan with contrast was done and demonstrated a telescoping of the small bowel in at least 3 areas with a target sign appearance with no signs of obstruction that suggested small-bowel intussusception. Following this imaging result, the patient was admitted under general surgery and kept nothing by mouth. The surgical team approached the gastroenterology team for push enteroscopy with biopsy which the patient refused. Further investigation included TTG antibodies; IgA and IgG were significantly elevated, 4,965.5 CU and $431.9 \mathrm{CU}$, respectively (reference: $<19.9 \mathrm{CU}$ ). The diagnosis of CD leading to adult intussusception was made. The patient
\end{abstract}

Our case has been presented poster presentation in the United European Gastroenterology (UEG) Week in 2021. 
was advised to start a gluten-free diet. During the hospital stay, the patient had complete resolution of his symptoms and was discharged home. In summary, this case highlights the potential link between adult intussusception and CD. Adult intussusception is a rare condition that its underlying etiology should be meticulously investigated to expedite treatment and prevent unnecessary surgical intervention.

(c) 2022 The Author(s).

Published by S. Karger AG, Basel

\section{Introduction}

Celiac disease (CD) is a chronic immune-mediated small-intestinal disorder triggered by gluten ingestion elicited in individuals carrying HLA-DQ2 or HLA-DQ8 genotypes [1]. Classical manifestations include diarrhea, abdominal pain, and weight loss. However, CD can present with anemia, osteoporosis, or arthralgia. Intussusception is a process where one segment of bowel invaginates into the lumen of an adjacent segment. Intussusception is a rare clinical condition in adults [2]. Despite that, the association between intussusception and CD has been descried previously, and it is still not widely recognized. Here, we report a case of adult intussusception as the first manifestation of CD.

\section{The Case History}

A 28-year-old, nonalcoholic and nonsmoker, previously healthy man presented to the emergency department with 1 week history of diffuse intermittent dull abdominal pain which was gradually and progressively exacerbated by food intake, especially wheat products, with no reliving factors associated with abdominal distension, nausea, vomiting, diarrhea, and $7 \mathrm{~kg}$ weight loss. He denied fever, night sweat, passage of fresh blood per rectum, having blackish tarry stool, or yellowish discoloration of the sclera. No history of skin rash or joint pain was also observed. He had no history of similar attack before and no significant family history. In addition, surgery or drug history was negative. He came back from Pakistan 2 months ago.

The physical exam was remarkable for thin body habitus and no lymphadenopathy, with normal bowel sounds and a soft nontender abdomen. No organomegaly could be observed. A digital rectal exam showed no rectal masses, hemorrhoids, or gross blood.

Laboratory testing was significant for hypochromic microcytic anemia, abnormal liver function (AST $214 \mathrm{IU} / \mathrm{L}$ [reference: $<50 \mathrm{IU} / \mathrm{L}$ ], ALT $217 \mathrm{IU} / \mathrm{L}$ [reference: <40 IU/L], and alkaline phosphatase 183 IU/L [reference: 40-129 U/L]), with a normal total bilirubin level and INR 2.9. Investigation of the elevated liver enzymes revealed negative autoimmune and viral serology. His workup for infectious causes of diarrhea was negative. An abdominal radiograph showed distended gas-filled small- and large-bowel loops. An abdominal sonogram revealed a normal liver with patent vasculature, an evidence of thickened loop of bowel with features suggestive of intussusception with a fluid-filled dilated bowel.

An abdominal computed tomography (CT) scan with IV contrast was done and demonstrated a telescoping of the small bowel in at least 3 areas with a target sign appearance (shown in Fig. 1,2) with no free fluid presented or enlarged lymph nodes and no signs of obstruction suggested of small bowel intussusception. Following this imaging result, the patient was admitted under general surgery and kept nothing by mouth (NPO). The surgical team approached the gastroenterology team for push enteroscopy with biopsy which the patient refused.

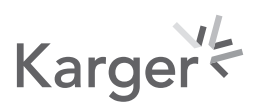


Fig. 1. Abdominal CT scan with IV contrast, axial section showing a multiple telescoping appearance of the small bowel with a classical target sign (white arrows).

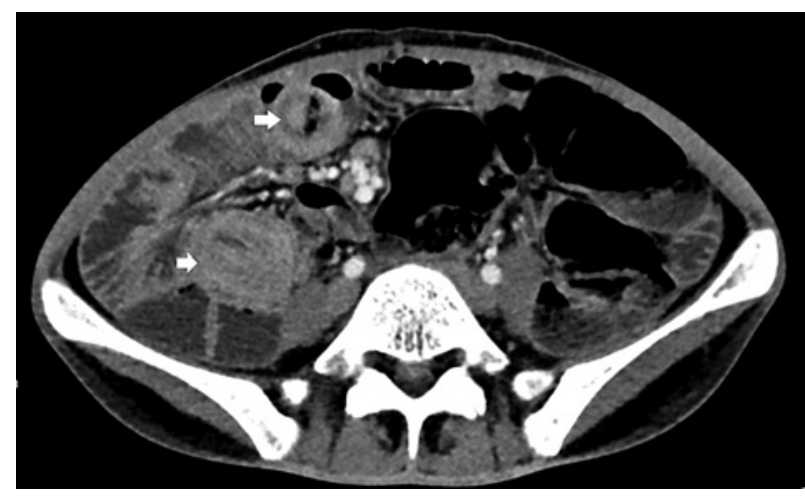

Fig. 2. Abdominal CT scan with IV contrast, sagittal section showing invaginated 224 mesenteric fat and vessels (white arrows).

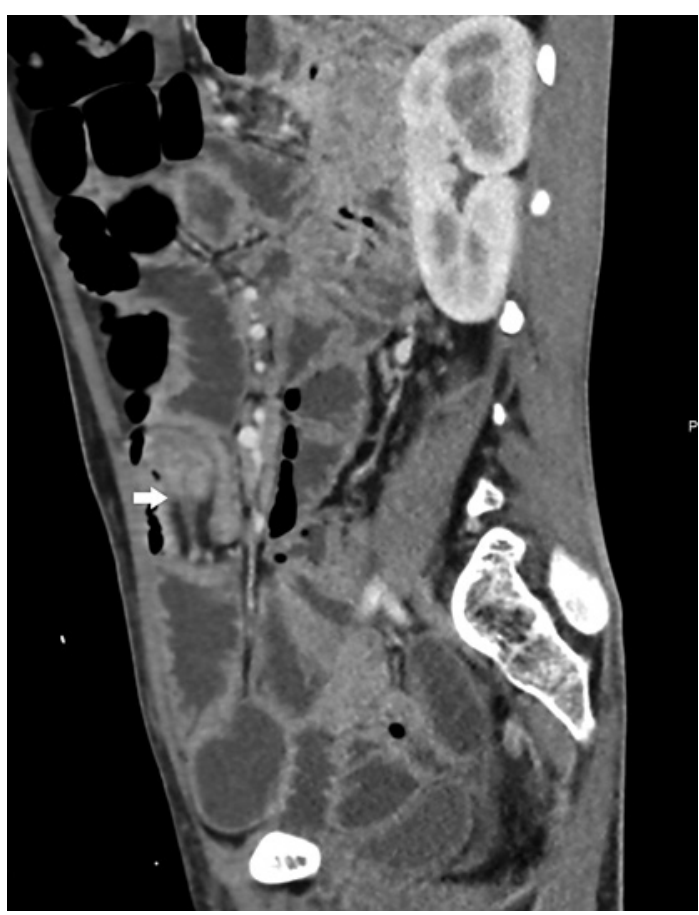

Further investigation included (tissue transglutaminase [TTG]) antibodies IgA and IgG being significantly elevated, 4,965.5 CU and 431.9 CU, respectively (reference: $<19.9 \mathrm{CU}$ ). The diagnosis of CD leading to adult intussusception was made.

The patient was advised to start a gluten-free diet. During the hospital stay, the patient had complete resolution of his symptoms and was discharged home. The patient traveled back to his home country for further evaluation.

\section{Discussion}

Adult intussusception remains a rare condition. It can be classified by underlying etiology as primary or idiopathic that occurs in the absence of a lead point, whereas in the secondary, a lead point is identified [2]. In a cohort of 58 surgically proven adult intussusception cases, $90 \%$ of cases were thought to be secondary to structural lesions [3]. CD stands out as least 
likely to be associated with intussusception. We are reporting a case of transient small-bowel intussusception, reveling CD in early adulthood life.

The association between CD and idiopathic intussusception has first been demonstrated in 1968 by Ruoff et al. [4]. Theories of adult intussusception development in CD are thought to invagination of one segment bowel loop into another due to dilated flaccid loops, disturbing normal peristaltic waves [5]. Sanders et al. [6] found that 4.9 years is the mean time from the symptoms onset to diagnosis of CD.

Nausea, vomiting, change in bowel habit, and abdominal distension are all features of intussusception in adults. Nowadays, intussusception is diagnosed by CT. The classic CT scan finding is the "target" sign or "sausage-shaped" soft-tissue mass [7]. In a case series involving 28 CT scans of CD adult patients who were reviewed, Tomei et al. [8] reported that intussusception was observed in up to $21 \%$. However, other CT scan findings have been reported in CD such as small-bowel folds pattern abnormalities, intestinal dilatation, bowel wall thickness, and lymphadenopathy [8].

Different etiological factors play a rule in adult intussusception. Among them are organic lesions such as inflammatory bowel disease, Meckel's diverticulum, benign or malignant neoplastic lesions, and iatrogenic or less commonly occurring CD [9]. Clinical, serological, and histological features are needed to diagnose CD. Anti-TTG sensitivity and specificity are 98.6 and $91 \%$, respectively [10].

Our patient presented with nonspecific gastrointestinal symptoms followed by CT scan imaging, confirming the diagnosis of adult intussusception with significantly elevated antiTTG. In view of patient preference, endoscopic evaluation was not performed. Gluten-free diet was initiated, and the patient improved conservatively without the need for surgical intervention.

In conclusion, this case highlights the potential link between adult intussusception and CD. Adult intussusception is a rare condition that its underlying etiology should be meticulously investigated to expedite treatment and prevent unnecessary surgical intervention.

\section{Acknowledgments}

The authors would like to thank the patient for his contribution.

\section{Statement of Ethics}

Written informed consent was obtained from the patient for publication of this case report and any accompanying images. Ethics approval for case report was not required. This has been approved by the Research and Ethics Committee in Sheikh Shakhbout Medical City.

\section{Conflict of Interest Statement}

The authors declare that they have no conflicts of interest.

\section{Funding Sources}

The authors declare that there was no funding for this research.

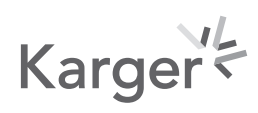


AlAhmad and Almessabi: Transient Small-Bowel Intussusception Unmasking Celiac Disease

\section{Author Contributions}

M.A. wrote the case and reviewed the literature. A.A. reviewed the manuscript and revised it.

\section{Data Availability Statement}

The data used to support this case is available within the article. Further inquiries can be directed to the corresponding author.

\section{References}

1 Fasano A, Catassi C. Clinical practice. Celiac disease. N Engl J Med. 2012 Dec 20;367(25):2419-26.

2 Wang N, Cui XY, Liu Y, Long J, Xu YH, Guo RX, et al. Adult intussusception: a retrospective review of 41 cases. World J Gastroenterol. 2009 Jul 14;15(26):3303-8.

3 Azar T, Berger DL. Adult intussusception. Ann Surg. 1997 Aug;226(2):134-8.

4 Ruoff M, Lindner AE, Marshak RH. Intussusception in sprue. Am J Roentgenol Radium Ther Nucl Med. 1968 Nov; 104(3):525-8.

5 Briggs JH, McKean D, Palmer JS, Bungay H. Transient small bowel intussusception in adults: an overlooked feature of coeliac disease. BMJ Case Rep. 2014 Jun 20;2014:bcr2013203156.

6 Sanders DS, Hurlstone DP, Stokes RO, Rashid F, Milford-Ward A, Hadjivassiliou M, et al. Changing face of adult coeliac disease: experience of a Single University Hospital in South Yorkshire. Postgrad Med J. 2002 Jan; 78(915):31-3.

7 Gayer G, Apter S, Hofmann C, Nass S, Amitai M, Zissin R, et al. Intussusception in adults: CT diagnosis. Clin Radiol. 1998 Jan;53(1):53-7.

8 Tomei E, Diacinti D, Marini M, Mastropasqua M, Di Tola M, Sabbatella L, et al. Abdominal CT findings may suggest coeliac disease. Dig Liver Dis. 2005 Jun;37(6):402-6.

9 Marinis A, Yiallourou A, Samanides L, Dafnios N, Anastasopoulos G, Vassiliou I, et al. Intussusception of the bowel in adults: a review. World J Gastroenterol. 2009 Jan 28;15(4):407-11.

10 Volta U, Fabbri A, Parisi C, Piscaglia M, Caio G, Tovoli F, et al. Old and new serological tests for celiac disease screening. Expert Rev Gastroenterol Hepatol. 2010 Feb;4(1):31-5. 